\title{
Zum 60. Geburtstag von Ass. Prof. Dipl.-Ing. Dr. mont. Gerhard Mayer
}

\author{
Eva-Maria Kern
}

Univ. d. Bundeswehr, München, Deutschland

Online publiziert 8. Januar 2020

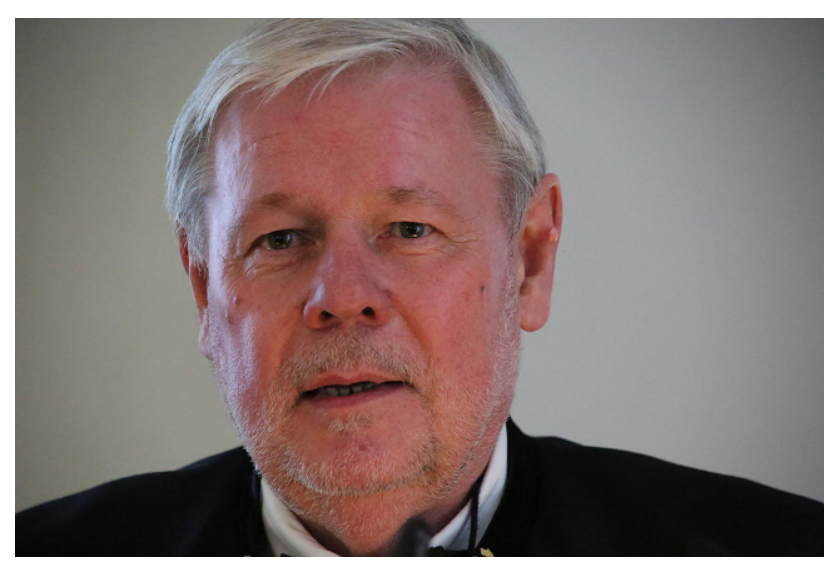

Sehr geehrter Herr Assistenzprofessor Mayer, lieber Gerhard,

sehr geehrter Herr Professor Wagner,

sehr geehrter Herr Vizerektor Moser,

Magnifizenz, (im Kontext der Wucht der 200 Jahre unserer Jubilare bitte ich um Nachsicht für die protokollarisch nicht ganz korrekte Reihenfolge der Begrüßung),

meine sehr geehrten Damen und Herren,

es ist mir eine ausgesprochene Freude und große Ehre zugleich, Ihnen in meiner Laudatio mit Herrn Assistenzprofessor Dipl.-Ing. Dr. mont. Gerhard Mayer einen ganz besonderen Menschen, mit dem mich mehr als 25 Jahre Freundschaft verbinden, näherbringen zu dürfen.

Eine Laudatio zu verfassen, stellt eine nicht zu unterschätzende Herausforderung dar. Ist es hierfür doch erforderlich, das im Allgemeinen etwas diffuse Gefühl der Verbundenheit mit einem Menschen und die persönliche Wertschätzung, die man demselben entgegenbringt, so zu kon-

Univ.-Prof. Dr.-Ing. habil. Dr. mont. E.-M. Kern, MBA ( $₫)$ Univ. d. Bundeswehr

München, Deutschland kretisieren und zu präzisieren, dass diese in Worte zu fassen sind.

Ausgehend von eigenen Gedanken habe ich diesen Versuch unternommen und wurde dabei auch durch liebe Wegbegleiter von Gerhard Mayer unterstützt, bei denen ich mich an dieser Stelle ganz herzlich bedanke. Nennen möchte ich hier insbesondere Frau Felser, Herrn Ass. Prof. Pilgram und Herrn Dipl.-Ing. Tscharf. Etwaige Fehlinterpretationen ihrer Beiträge gehen selbstverständlich allein zu meinen Lasten. Somit sollte auch nach dieser Laudatio der weiteren ungetrübten und friedvollen Zusammenarbeit der Genannten mit unserem Jubilar nichts entgegenstehen.

Meine Damen und Herren, ich werde in den nächsten Minuten unseren Jubilar aus zwei unterschiedlichen Perspektiven würdigen. Zunächst wie es den akademischen Gepflogenheiten entspricht - mit Blick auf seinen Lebenslauf und seine Funktionen als Forscher, Lehrer und Geschäftsführer des BVÖ. Abrunden werde ich das Bild mit einer Betrachtung des Menschen und Freundes.

\section{Zum Curriculum Vitae}

Gerhard Mayer wurde am 14. November 1959 in Ybbs an der Donau, also in Niederösterreich, geboren.

Nach der Volksschule in Persenbeug besuchte er das Gymnasium in Wieselburg. Dass sein Schulkollege ein ehemaliger österreichischer Bundeskanzler war, hat meines Wissens seinen weiteren Lebensweg nicht beeinflusst.

Unmittelbar nach der Matura kam das Militär - und dann begann mit dem Studium des Markscheidewesens auch schon Gerhard Mayers Geschichte in Leoben an unserer Alma Mater. Die Wissenschaft hat inn so begeistert, dass er ihr treu blieb und 1992 mit sehr gutem Erfolg zum Doktor der montanistischen Wissenschaften promoviert wurde - und zwar mit der Dissertation "Beobachtung eines Senkungstroges mit gravimetrischen und markscheiderischen Methoden im tertiären Kohlenbergbau“. 


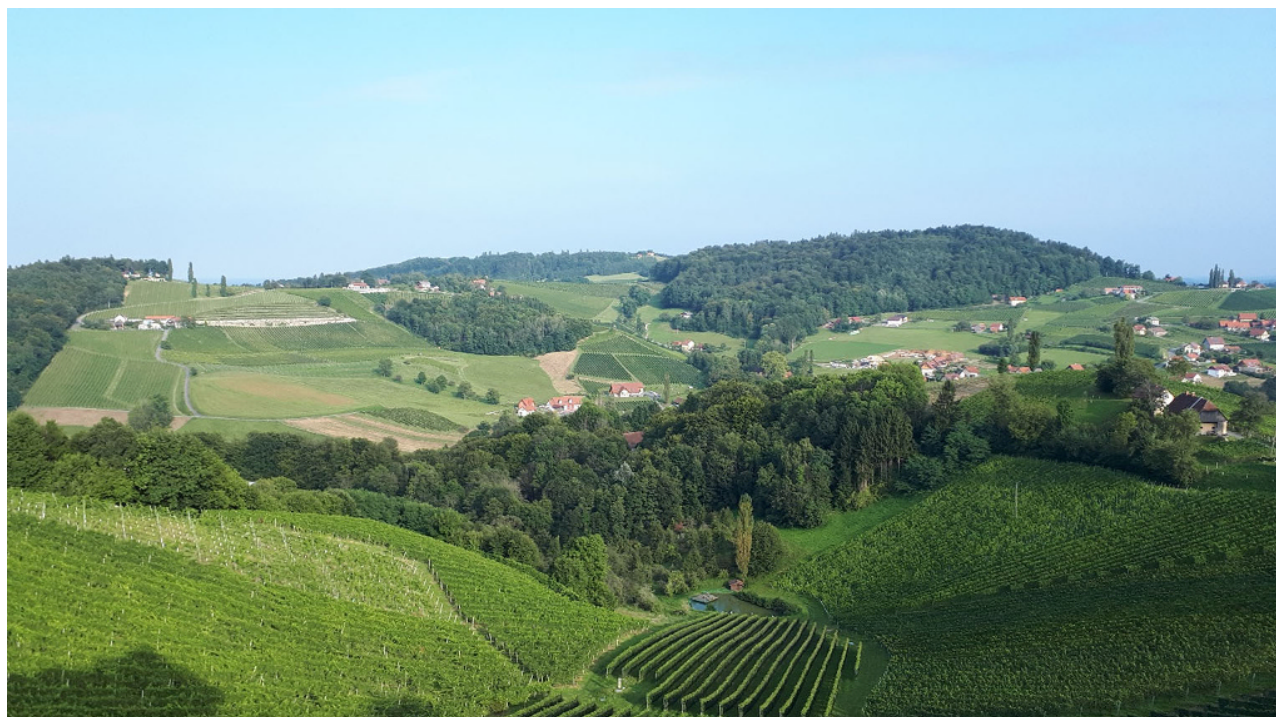

Bereits neben seinem Studium war er freier Mitarbeiter im Ingenieurbüro seines von ihm sehr verehrten akademischen Lehrers Prof. Dr. mont. Eduard Czubik, an dessen Institut für Markscheide- und Bergschadenkunde er auch Assistent war.

In den Jahren 1986 und 1994 hat er jeweils für rund 2 Monate an den Forschungsreisen dieses Institutes zur Erforschung rezenter Erdkrustenbewegungen in Island teilgenommen.

Seit 1999 ist er Assistenzprofessor an der Montanuniversität Leoben, zuerst am Institut für Markscheide- und Bergschadenkunde und seit 2002 am Lehrstuhl für Bergbaukunde, Bergtechnik und Bergwirtschaft.

Aus seiner Forschungstätigkeit sind 65 wissenschaftliche Publikationen hervorgegangen, die er als Autor oder Koautor verfasst hat.

Gerhard Mayer verfügt auch über langjährige Erfahrung in der wissenschaftlichen Lehre. Nicht nur seit 1988 an der Montanuniversität Leoben, sondern seit 2009 bzw. 2012 auch an der TU Bergakademie Freiberg und an der RWTH Aachen.

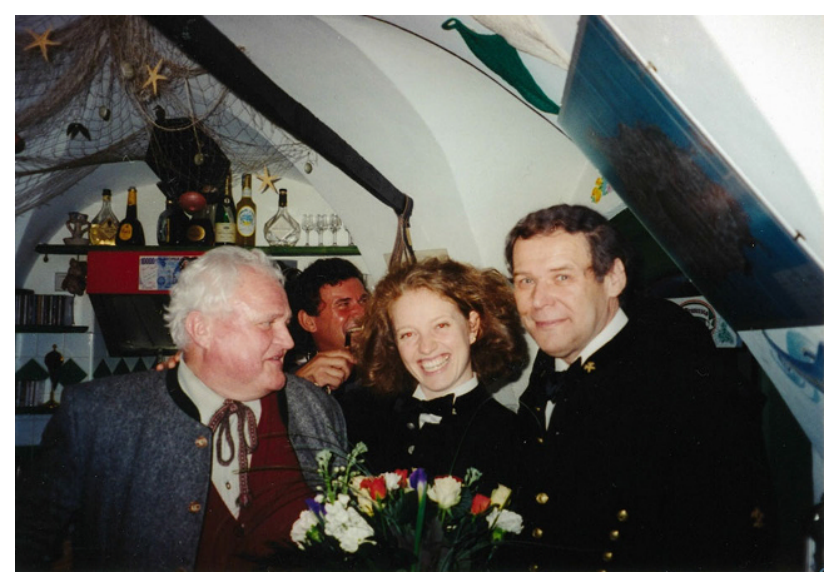

Alte Freunde 1: Czubik, Aubell, Kern, Kepplinger
Darüber hinaus ist er seit 1997 Geschäftsführer des Bergmännischen Verbandes Österreichs. Dieses Amt füllt er mit Leib und Seele besonders engagiert aus, da ihm der BVÖ sehr am Herzen liegt. Sein erfolgreiches Wirken spiegelt sich u. a. in der beachtlichen Zahl der Mitglieder des BVÖ wider.

Als weitere wesentliche Funktionen unseres Jubilars sind zu nennen:

- Herausgebervertreter bzw. Editor-in-Chief der Berg- und Hüttenmännischen Monatshefte,

- seine Mitgliedschaft im Vorstandsbeirat des Deutschen Markscheider-Vereins e. V. und

- sein Amt als Vertreter Österreichs im Präsidium der Internationalen Gesellschaft für Markscheidewesen.

Damit nicht genug - Gerhard Mayer engagiert sich auch für jene, mit denen es das Leben nicht so gut gemeint hat und die darüber hinaus durch das soziale Netz fallen: Schon als "Junger", d. h. bis 40, war er Mitglied beim "Round Table". Danach und bis heute wirkt er beim "Club $41^{\prime \prime}$ - in beiden

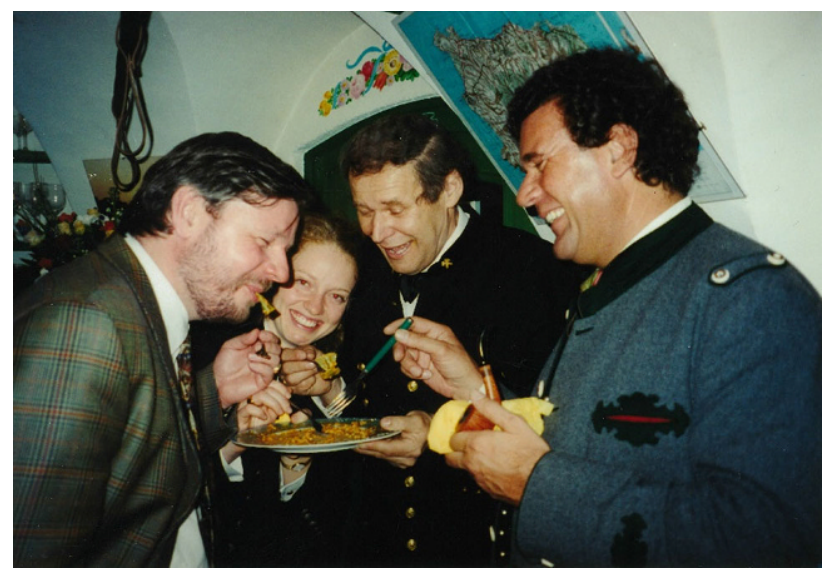

Alte Freunde 2: Mayer, Kern, Kepplinger, Aubell 


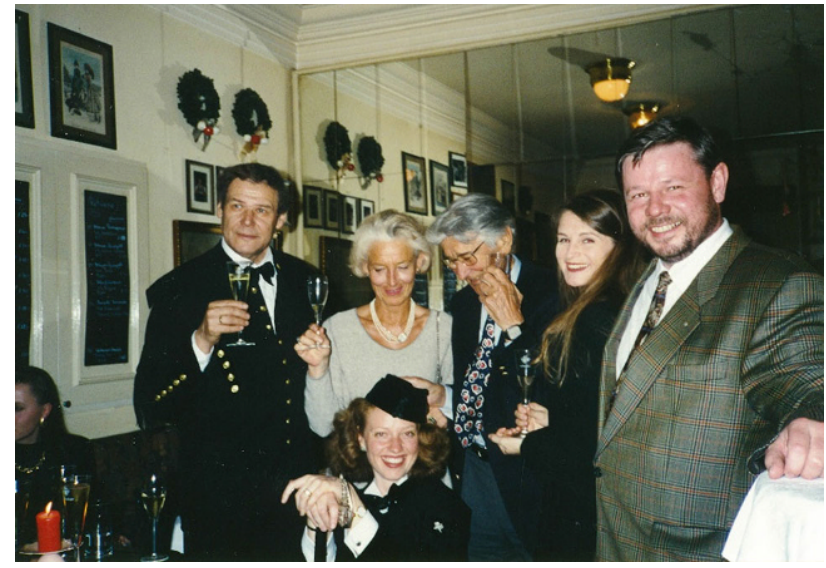

Promotionsfeier Kern

Serviceclubs hatte er u. a. die Funktion des Präsidenten inne.

Lassen Sie mich nun folgende Details aus dem gerade vorgestellten Curriculum ein bisschen mit Leben füllen: seine Lehrtätigkeit und einen kleinen Ausschnitt aus seinem Arbeitsalltag.

Was kennzeichnet Gerhard Mayer in seiner Funktion als akademischer Lehrer? Er verfügt über das Talent, Inhalte begreifbar zu machen und diese bleibend (heute würde man sagen: nachhaltig) zu vermitteln. Das gelingt ihm auch nachdrücklich dadurch, dass er nicht auf Teufel komm heraus den gesamten Stoffumfang zu präsentieren sucht, sondern sich auf das aus seiner Sicht Wesentliche konzentriert. Besonders engagiert er sich für die bei den Studierenden beliebten Feldübungen, insbesondere am Erzberg. Allerdings scheint er der Sturm- und Drangzeit dahingehend entwachsen zu sein, dass - so wurde mir berichtet im Rahmen der Feldübungen nicht mehr am Erzberg mehrere Tage campiert werden muss.

Wichtig zu erwähnen ist, dass unser Jubilar immer auf der Seite der Studierenden und Doktoranden ist und war. Ein echter Fürsprecher und oft auch persönliches Sprachrohr nach außen und innerhalb des Institutes - immer zum Entgegenkommen bereit. Im Gegenzug dazu verlangt er

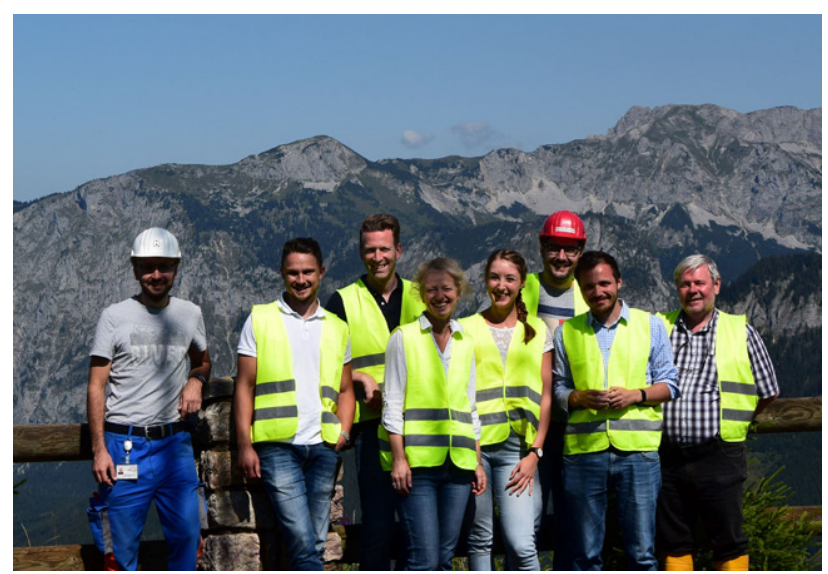

Exkursion des Lehrstuhls Prof. Kern am „Erzbergspitz”

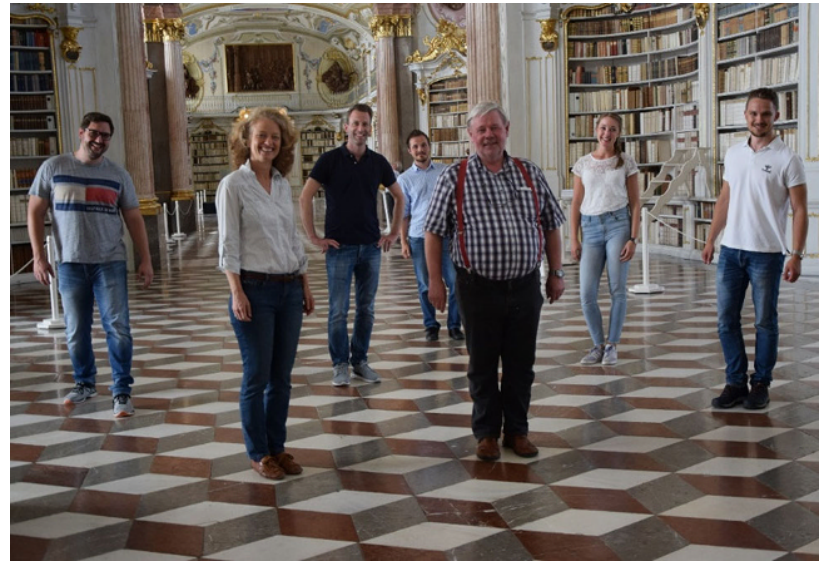

Exkursion des Lehrstuhls Prof. Kern Stiftsbibliothek Admont

Engagement und Verlässlichkeit und fordert Leistung auch ein. Darüber hinaus schätzt er auch den kommunikativen Austausch mit dem akademischen Nachwuchs, den er auf diversen Veranstaltungen pflegt - und er ist auch nicht der Erste, der nach Hause geht.

Arbeitstechnisch scheint Gerhard Mayer eher eine Lerche als eine Nachtigall zu sein. Dem Hörensagen nach schätzt er - sehr atypisch für unseren Berufsstand - die akademische Morgendämmerung.

Zu frühmorgendlicher Stunde begibt er sich, stets gut gelaunt und ausgerüstet mit seinem Jausensackerl an die Uni, wo er als erstes, vor allem im Winter, den er besonders mag, seiner Sekretärin einen Kälteschock versetzt, indem er das Wort Stoßlüftung besonders ernst nimmt. Nach der Morgenbesprechung mit Frau Felser begibt er sich zum sogenannten Markscheiderstammtisch zu Ass. Prof. Pilgram. Dort werden als Vorbereitung auf den Arbeitstag fachliche und - so eine vertrauliche Quelle -zuweilen auch außerfachliche Themen, selbstverständlich stets wissenschaftlich, erörtert.

Während sich die beiden genannten Besprechungen dadurch auszeichnen, dass sie zu definierten Zeiten an definierten Orten stattfinden, gestaltet sich im weiteren Tagesverlauf die Gesprächsanbahnung mit unserem Jubilar zuweilen als herausfordernd. Da er, wie es sich für seine Doppelfunktion als Assistenzprofessor und Geschäftsführer des BVÖ gehört, über zwei Büros verfügt, beginnt für so manchen Gesprächswilligen eine spannende Suche. Wie ich gehört habe, hält sich Gerhard Mayer zumeist nicht in dem Büro auf, in dem man inn gerade vermutet.

Meine Damen und Herren, es gibt für einen Chef wohl kein größeres Lob, als wenn über ihn gesagt wird, dass das, was er tut, immer Hand und Fuß hat, er sich selbst zurück und nicht so wichtig nimmt, die Fäden zieht, sich um alles verlässlich kümmert und dabei immer das große Ganze im Blick hat. Dem ist nichts mehr hinzuzufügen, das spricht für sich und unseren Jubilar. Etwaige negative Energien scheinen sich im nahezu täglichen Messerschleifen zu kulminieren und abzubauen. Wahrscheinlich ist dies aber eine Überinterpretation einer zutiefst männlichen Tätigkeit und dient letztendlich einfach dem Zweck eines einwandfrei geschnittenen Jausenspecks. 
Nun, meine Damen und Herren, darf ich die beruflichamtliche Ebene verlassen.

Die Darstellung eines akademischen Lebenslaufes rechtfertigte bisher die Ausführungen in der dritten Person die ich aber jetzt, bei der Beschreibung des Menschen und Freundes gerne verlassen möchte.

Lieber Gerhard, ein Bild sagt mehr als tausend Worte und hilft, komplexe Sachverhalte kompakt zu verdeutlichen. Und so werde ich meine Beschreibung von Dir auch mit einem Bild beginnen. Als Montanistin habe ich natürlich zuerst im Umfeld des Bergbaus, der Markscheidekunde oder aber der Geologie gesucht. Mangels Fachkompetenz (wie Du weißt, bin ich Kunststofftechnikerin) allerdings ausgesprochen dilettantisch. Und so konnte mir Reinfried Pilgram auch problemlos ausreden, Dich mit einem Theodoliten, dem Erzberg oder einem Geysir zu vergleichen, auch wenn Du in Island warst.

Hätte ich nur ein klein wenig quer und weniger fachlich gedacht, wäre es mir sofort klar gewesen.

Lieber Gerhard, das ist mein Bild für Dich. Es ist der Landstrich, den Du besonders magst: die Südsteiermark.

Auf den ersten Blick ruhig, beständig und auch bodenständig, freundlich und offen - nicht laut, nicht aufdringlich, keine großen Extreme, weder schroffe Gipfel noch tiefe Täler. Steirische Hügellandschaft, friedlich und beschaulich. Und schaut man sich die Gegend näher an, sieht man ihre Details und ihre Vielfalt.

Lieber Gerhard, diese Landschaft passt nicht nur gut zu Dir, sondern bietet Dir auch ganz viel von dem, was Dich zufrieden und glücklich macht. Als ausgesprochener Genussmensch liebst Du gutes Essen, das Leib und Seele zusammenhält - und weißt auch einen guten Tropfen stets zu schätzen. Und die Köstlichkeiten, die nicht gleich verzehrt werden, nimmst Du mit nach Hause und verarbeitest sie bei Deinem Lieblingshobby Kochen. Deine selbst gesammelten Schwammerl bereichern Deine Gerichte. Das Handwerk des Schwammerlsuchens scheinst Du bestens zu beherrschen und bis dato auch unbeschadet überstanden zu haben.

Lieber Gerhard, zum Abschluss hab' ich Dir aus alten Zeiten ein paar Erinnerungen mitgebracht. Von Professor Czubik, den wir beide sehr verehrt haben, von unserer sog. "Stammrunde“, wir hatten ja keinen Tisch, mit Dr. Eginhard Aubell und meinem Doktorvater, Professor Kepplinger. Wie viele Stunden wir im Francesco verbracht, was genau wir geredet, gegessen und getrunken haben, ich kann es nicht mehr sagen. Ich weiß nur, es waren schöne Zeiten.

Schöne Zeiten sind es auch jetzt, in der Gegenwart - wo Du meinen leobenunkundigen Assistenten und mir einen unvergesslichen Tag in der Region bereitet hast.

Danke für Deine Freundschaft, Beständigkeit und Verlässlichkeit - und danke besonders dafür, dass Du auch als Markscheider in der Kunststofftechnikerin eine Montanistin gesehen hast. Die Barbara und das silberne Berghäckel werden mich immer daran erinnern!

Lieber Gerhard, in Vertretung für all jene Menschen, denen Du etwas bedeutest, sei es privat oder beruflich wünsche ich Dir aus ganzem Herzen zu Deinem bevorstehenden 60er das Allerbeste. Vor allem Gesundheit und Glück.

Bitte bleib genau so wie Du bist!

Ad multos annos,

Glück Auf!

Vizepräsidentin Univ.-Prof. ${ }^{\text {in }}$

Dr.-Ing. habil. Dr. mont. Eva-Maria Kern, MBA 